\title{
LA AGENCIA INTERNACIONAL PARA LAS ENERGÍAS RENOVABLES: PROMESA INSTITUCIONAL ANTE LOS DESAFÍOS ENERGÉTICOS DEL SIGLO XXI
}

\author{
Miguel Á. Elizalde CARranza \\ Profesor de Derecho Internacional Público \\ Universitat Pompeu Fabra \\ miguelangel.elizalde@upf.edu
}

Recibido: 14 de diciembre de 2015 / Aceptado: 27 de mayo de 2016

RESUMEN: La nota describe los antecedentes, la estructura institucional, el mandato y las actividades de la Agencia Internacional para las Energías Renovables (IRENA). La nota también incluye un comentario sobre los logros de los primeros años de actividades de la IRENA y de los retos con vistas al futuro.

RESUM: La nota descriu els antecedents, l'estructura institucional, el mandat i les activitats de l'Agència Internacional per a les Energies Renovables (IRENA). La nota també inclou un comentari sobre els assoliments dels primers anys d'activitats de la IRENA i dels reptes de cara al futur.

ABSTRACT: The note describes the antecedents, the institutional structure, the mandate and the activities of the International Agency for the Renewable Energies (IRENA). The note also includes a comment on the attainments of the first years of activities of IRENA, and about its future challenges.

PALABRAS CLAVE: Energías renovables - Gobernanza energética - Desarrollo sostenible — IRENA 
PARAULES CLAU: Energies renovables - Governança energètica Desenvolupament sostenible — IRENA

KEYWORDS: Renewable energy — Energy Governance — Sustainable Development - IRENA

SUMARIO: I. Introducción. II. Antecedentes y aspectos generales. 1. Antecedentes. 2. Aspectos generales de la IRENA. III. Las actividades, iniciativas y proyectos de la Agencia. 1. Descripción general de las actividades de la Agencia. 2. Principales iniciativas y proyectos de la Agencia. 2.1. La evaluación del potencial para el despliegue de energías renovables. 2.2. La función de nodo de confluencia para las energías renovables. 2.3. El Portal de Conocimiento sobre Energías Renovables. 2.4. Iniciativas para promover las inversiones y el crecimiento económico. IV. Logros y retos futuros de la Agencia. 1. Los logros. 1.1. Ser una organización internacional independiente. 1.2. Haber alcanzado una membrecía prácticamente universal. 1.3. Resultados con alto potencial económico a bajo coste. 1.4. Ha sabido evitar la parálisis sobre las acciones globales sobre el cambio climático. 2. Los retos de la Agencia. 2.1. Posicionarse como centro de información de excelencia a nivel global. 2.2. Lograr movilizar el apoyo de países clave que aún no son miembros. 2.3. Evitar su instrumentalización. V. Conclusiones. VI. Fuentes y bibliografía.

\section{INTRODUCCIÓN}

La energía ocupa hoy en día uno de los principales lugares en la agenda internacional dadas sus profundas implicaciones económicas, de seguridad y para el medio ambiente ${ }^{1}$. Por lo tanto, la comunidad internacional se adentra en el siglo XXI enfrentando el desafío de movilizar la cooperación internacional para favorecer la buena gobernanza global en materia de energía. En gran medida, las políticas energéticas que se adopten en un futuro inmediato serán las que determinen hasta qué punto el desarrollo y el bienestar de la comunidad internacional y de sus futuras generaciones quedarán hipotecados por el cambio climático. En ese sentido, los principales expertos indican que cada vez es más urgente reducir de forma sustancial y sostenida las emisiones de $\mathrm{CO}_{2}$ para tener opciones de lograr los objetivos de la Convención Marco de las Naciones Unidas sobre el Cambio Climático (CMNUCC) y de su Protocolo de Kioto ${ }^{2}$.

\footnotetext{
${ }^{1}$ Véase, en general, ESCRIBANO, G., "Fragmented Energy Governance and the Provision of Global Public Goods", Global Policy, vol. 6, Issue 2, 2015, pp. 97 y ss.; CORRELJÉ, A. y VAN DER LINDE, C., "Energy Supply Security and Geopolitics: A European Perspective", Energy Policy, vol. 34, núm. 5, 2006, pp. 532-543; y ABBOTT, K. W., "The Transnational Regime Complex for Climate Change", Environment and Planning C: Government and Policy, vol. 30, núm. 4, 2012, pp. 571-590.

2 IPCC, "Resumen para responsables de políticas", EDENHOFER, O. et al. (eds.), Cambio climático 2014: Mitigación del cambio climático, Cambridge University Press, Cambridge, Reino Unido y Nueva York, 2015, p. 6.
} 
En este escenario, el incremento de la producción, uso y comercialización de las energías renovables se presenta globalmente como un reto, pero también como una ventana de esperanza, ya que constituyen energía limpia que puede producirse localmente y que crea nuevas oportunidades de inversión, comerciales y laborales. Aunque las inversiones en el sector aumentan progresivamente, aún existen importantes lagunas de información que dificultan el aprovechamiento del potencial de las energías renovables; por ejemplo, existe poca información en relación con los costes y las condiciones de los distintos países para el despliegue de estas fuentes de energía ${ }^{3}$.

En general, la gobernanza de la energía está fragmentada. Existe una gran variedad de instituciones con diferencias en sus mandatos y ámbitos materiales y geográficos de influencia, membrecía, presupuesto, intereses, etc. ${ }^{4}$ Por ello, resulta significativa la creación, en 2009, de la Agencia Internacional para las Energías Renovables (IRENA, por sus siglas en inglés $)^{5}$ —en lo sucesivo, la Agencia-, cuyo objetivo es crear y diseminar conocimiento que facilite un mayor despliegue y aprovechamiento de las energías renovables sostenibles. Pese a ser una institución con un presupuesto muy reducido, está diseñada para contribuir a los esfuerzos internacionales ante los desafíos energéticos del siglo XXI y para facilitar la transición hacia fuentes sostenibles.

Esta nota intenta contribuir a dar notoriedad a la IRENA, la primera organización internacional creada en el siglo XXI ${ }^{6}$. Para lograr este objetivo, la nota se estructura en

\footnotetext{
3 IRENA. (2014, 18 de enero). Work Programme and Budget for 2014-2015: Report of the Director General. IRENA Doc. A/4/3, p. 16. Recuperado el 23 de mayo de 2016, de https://www.irena.org/DocumentDownloads/A_4_3_WPB.pdf [En lo sucesivo, Work Programme and Budget 2014-2015]

${ }^{4}$ LONG, ANDREW, “Complexity in Global Energy-Environment Governance”, en Minnesota Journal of Law, Science \& Technology, Vol. 15, 2014, pp. 1059-1115, p. 1080.

${ }^{5}$ Estatuto de la Agencia Internacional de Energías Renovables (IRENA), hecho en Bonn el 26 de enero de 2009, en vigor desde el 8 de julio de 2010 (1 de abril de 2011 para España). Se puede consultar en el BOE No. 75, 29 de marzo de 2011, p. 32574 y ss. [en lo sucesivo Estatuto IRENA]

${ }^{6}$ Los pocos estudios que existen han sido publicados en inglés: WRIGHT, G., "The International Renewable Energy Agency: A Global Voice for the Renewable Energy Era?", Renewable Energy Law and Policy Review, vol. 4, 2011, pp. 251-268; RÖHRKASTEN, S. y WESTPHAL, K., "IRENA and Germany Foreign Renewable Energy Policy: Aiming at Multilevel Governance and an Internationalization for the Energiewende?", SWP: Working Paper FG 8, 2013/01, septiembre de 2013; MEYER, T., "Global Public Goods, Governance Risk, and International Energy", Duke Journal of Comparative \& International Law, vol. 22, 2012, pp. 319-347; MEYER, T., "Epistemic Institutions and Epistemic Cooperation in International Environmental Governance", Transnational Environmental Law, vol. 2, núm. 1, 2013, pp. 15-44; URPELAINEN, J. y VAN DE GRAAF, T., "The International Renewable Energy Agency: A Success Story in Institutional Innovation?", International Environmental Agreements: Politics, Law and Economics, vol. 15, núm. 2, 2015, pp. 159-177; VAN DE GRAAF, T., "Fragmentation in Global Energy Governance: Explaining the Creation of IRENA", Global
} 
cinco apartados. El primer apartado contiene esta introducción; el segundo describe los antecedentes y algunos aspectos generales; el tercero analiza las actividades, las iniciativas y los proyectos de la institución; el cuarto se ocupa de los logros y retos futuros de la IRENA; el quinto, y último, contiene las conclusiones.

\section{ANTECEDENTES Y ASPECTOS GENERALES}

\section{Antecedentes}

La idea inicial de H. Scheer, padre ideológico y principal impulsor de la IRENA, fue que la Agencia se creara bajo el paraguas de las Naciones Unidas, pese a que en 1981, en la I Conferencia Internacional de las Naciones Unidas sobre Fuentes de Energía Nuevas y Renovables, celebrada en Nairobi, Kenia, ya se había discutido sin éxito esta posibilidad $^{7}$. Así, en 1990 presentó en la sede de las Naciones Unidas en Nueva York una propuesta para crear la Agencia Internacional para la Energía Solar (AIES) ${ }^{8}$. Sin embargo, el Comité encargado de preparar la Conferencia de Río sobre el Medio Ambiente y el Desarrollo (CNUMAD), prevista para 1992, rechazó la iniciativa ${ }^{9}$. En general, las energías renovables no recibieron suficiente impulso en la Conferencia de Río ni tampoco en la Cumbre Mundial sobre el Desarrollo Sostenible de 2002, celebrada en Johannesburgo ${ }^{10}$. La desilusión generada por la falta de progreso en el marco de las Naciones Unidas llevó a los promotores de la IRENA a buscar alternativas que prescindieran del consenso como condición para avanzar hacia el establecimiento de la institución ${ }^{11}$.

Environmental Politics, vol. 13, núm. 3, 2013, pp. 14-33; RÖHRKASTEN, S. y WESTPHAL, K., "IRENA: Stay the Course", SWP Comments, núm. 37, noviembre de 2012, pp.1-4.

7 SCHEER, H., "Memorandum for the Establishment of an International Renewable Energy Agency, 2000", EUROSOLAR y WCRE (eds.), The Long Road to IRENA: From the Idea to the Foundation of the International Renewable Energy Agency, Ponte Press, Bochum, 2009, pp. 24-33.

${ }^{8}$ El 15 de enero de 1990 Scheer ya había presentado en Bonn, Alemania, un memorándum para proponer la creación de la Agencia Internacional de la Energía Solar.

${ }^{9}$ Entre las razones de este rechazo se encontraban la resistencia generalizada a las energías renovables, el deseo de la Agencia Internacional de la Energía (AIE) y de la Agencia Internacional de la Energía Atómica (AIEA) de evitar la competencia y la falta de voluntad de algunos países de crear nuevas instituciones al sentirse insatisfechos con el funcionamiento de las que ya existían. EUROSOLAR y WCRE (eds.), The Long... cit., p. 4.

${ }^{10}$ BUTLER, J., “The Second Earth Summit”, Geography Review, mayo de 2003, p. 4.

11 The Federal Government of Germany, The Case for an International Renewable Energy Agency (RENA), Preparatory Conference for the Foundation of IRENA, 10-11 de abril de 2008, p. 10. Muy crítico, Scheer escribió: "Proceder de manera unilateral incluso se puede convertir en un imperativo 
Alemania, en donde la idea de crear la Agencia había ido ganando apoyos en el sector público desde 1998, celebró una conferencia internacional sobre energías renovables en Bonn en 2004 (Renewables 2004). Sin embargo, contrariamente a la idea original, no se propuso la creación de la IRENA; algo que, por otro lado, fue la recomendación principal del Foro Parlamentario Internacional de Energías Renovables que tuvo lugar de forma paralela a Renewables 2004 y al que, por invitación del Bundestag alemán, asistieron representantes de 67 parlamentos diferentes ${ }^{12}$.

La coalición de democratacristianos y socialdemócratas que llegó al poder en 2005 en Alemania dio un mayor apoyo político a la iniciativa. El Gobierno alemán inició una campaña internacional, principalmente a través de consultas bilaterales, y en 2008 inició formalmente el proceso preparatorio para la creación de la IRENA ${ }^{13}$. En dos años la diplomacia alemana logró algo que en treinta años no se había podido alcanzar en el marco de las Naciones Unidas. Bastaron dos conferencias preparatorias, realizadas en el año 2008, una en Alemania y otra en España, para que la Agencia fuera establecida el 29 de enero de 2009 en la conferencia fundacional de Bonn, en la que participaron representantes de más de 120 países ${ }^{14}$. Las motivaciones para participar variaban según los países: algunos lo hacían al considerar que la Agencia podría facilitarles alcanzar objetivos de sus políticas internas sobre energías renovables; otros, porque tenían problemas de suministro energético; otro grupo, por las posibles oportunidades comerciales para sus empresas activas en el ámbito de la energía renovable o porque sus territorios poseían potencial para el despliegue de fuentes de energía renovable ${ }^{15}$. También se sospecha que algunos países ingresaron en la Agencia para intentar influir en el diseño de su agenda, en particular, para evitar injerencias en sus intereses o en los de otras organizaciones internacionales. Setenta y cinco Estados firmaron el Estatuto de la Agencia en la conferencia fundacional. El Estatuto entró en vigor el 8 de julio de

urgente cuando un método multilateral de acción se muestre como un fracaso evidente y no emerja ninguna solución satisfactoria". SCHEER, H., Autonomía Energética: La situación económica, social y tecnológica de la energía renovable, Icaria, Barcelona, 2009, p. 180.

12 EUROSOLAR y WCRE (eds.), "International Parliamentary Forum", The Long... cit., p. 70. International Parliamentary Forum on Renewable Energies. (2004, 2 de julio). The Challenges of the $21^{\text {st }}$ Century. Recuperado el 23 de mayo de 2016, de http://www.hermannscheer.de/en/images/stories/pdf/IPF04_Resolution_en.pdf.

${ }^{13}$ RÖHRKASTEN y WESTPHAL, “IRENA and Germany...," cit., pp. 7 y 8.

14 IRENA. (2015). Creation of IRENA. Recuperado el 23 de mayo de 2016, de http://www.irena.org/menu/index.aspx?mnu=cat\&PriMenuID=13\&CatID=30. Véase también: The Federal Government of Germany, The Case... cit.

${ }^{15}$ RÖHRKASTEN y WESTPHAL, "IRENA and Germany...", cit., p. 8. 
2010, lo que convirtió a la IRENA en la primera organización internacional creada en el siglo XXI.

\section{Aspectos generales de la IRENA}

La Agencia tiene su sede en Abu Dabi, Emiratos Árabes Unidos (EAU); es una organización internacional universal abierta a todos los miembros de las Naciones Unidas y a las organizaciones internacionales de integración económica ${ }^{16}$. Actualmente, la IRENA cuenta con 147 miembros ${ }^{17}$.

La Agencia tiene como misión la promoción e implantación generalizada y reforzada de todas las formas de energía renovable y su uso sostenible ${ }^{18}$. El Estatuto de la Agencia establece que deberá entenderse por "energías renovables" todas las formas de energía que provengan de fuentes renovables y que sean producidas de manera sostenible; y menciona que se incluyen, entre otras, la bioenergía, la energía geotérmica, la energía hidráulica, la energía marina, la energía solar y la energía eólica ${ }^{19}$. Dado que los impactos ambientales negativos de muchas de las energías renovables que se citan en el Estatuto son objeto de crítica por algunos $\operatorname{sectores}^{20}$, cabría establecer cómo se determina en este contexto si una energía renovable se produce de forma sostenible.

Por lo que se refiere a la estructura institucional, la Asamblea es el órgano supremo de la Agencia $^{21}$ y está integrada por representantes de todos los Estados miembros ${ }^{22}$. Salvo decisión en contrario, celebra sesiones periódicas en la sede de la IRENA una vez al año ${ }^{23}$. El Consejo es el segundo órgano más importante y está integrado por veintiún representantes de los Estados miembros elegidos con carácter rotativo por la Asamblea

\footnotetext{
${ }^{16}$ Artículo VI del Estatuto IRENA.

17 IRENA. (2016). Membership. Recuperado el 23 de mayo de 2016, de http://www.irena.org/menu/Index.aspx?mnu=Cat\&PriMenuID=46\&CatID=67.

${ }^{18}$ Artículo II del Estatuto IRENA.

${ }^{19}$ Ibidem, artículo III.

${ }^{20}$ PRING, G. et al., "The Impact of Energy on Health, Environment, and Sustainable Development", ZILlMAN, Donald N. et al. (eds.), Beyond the Carbon Economy: Energy in Transition, Oxford University Press, Nueva York, 2008, pp. 13-37.

${ }^{21}$ Artículo IX. A.1 del Estatuto IRENA.

${ }^{22}$ Ibidem, artículos IX. A.1 y. IX. B.

${ }^{23}$ Ibidem, artículo IX. B.
} 
para un período dos años $^{24}$. En la elección de los miembros del Consejo se debe garantizar la participación efectiva de los países desarrollados y en desarrollo, y un reparto geográfico justo y equitativo ${ }^{25}$. El Consejo se convoca cada seis meses y se reúne, salvo acuerdo en contrario, en la sede de la Agencia ${ }^{26}$. Además, cuenta con la Secretaría, compuesta por el director general, como órgano rector y director administrativo, y por el personal que resulte necesario. La designación del director general la realiza la Asamblea, previa recomendación del Consejo, por períodos de cuatro años, que podrán ser prorrogados en una ocasión ${ }^{27}$. Dentro de la estructura institucional también se encuentra el Centro de Innovación y Tecnología (CIT), aunque no es uno de sus órganos. El CIT fue establecido en Bonn como premio de consolación a Alemania al no habérsele otorgado la sede de la Agencia. El CIT se inauguró en octubre de 2011 y se presenta como un centro de excelencia en innovación y tecnología sobre energías renovables, con un enfoque práctico $^{28}$. Uno de sus objetivos principales es facilitar el acceso a la información relacionada con las patentes sobre energías renovables, de las que se considera que existen cerca de $215.000^{29}$. Además, elabora estudios sobre costes y eficiencia de las energías renovables, y sobre la viabilidad de la transferencia de tecnología ${ }^{30}$.

\section{LAS ACTIVIDADES, INICIATIVAS Y PROYECTOS DE LA AGENCIA}

Conviene iniciar diciendo que las actividades de la IRENA son de naturaleza epistémica. MEYER define la "cooperación epistémica" como la forma en la que los Estados organizan el proceso de crear conocimiento científico compartido pertinente para la cooperación ambiental, y considera como "instituciones epistémicas" las instituciones internacionales implicadas en crear conocimiento científico compartido ${ }^{31}$.

\footnotetext{
${ }^{24}$ Ibidem, artículo X. A.

${ }^{25}$ Idem.

${ }^{26}$ Ibidem, artículo X. B.

${ }^{27}$ Artículo XI. B del Estatuto IRENA.

${ }^{28}$ IRENA. (2016). Institutional Structure: Innovation and Technology Centre. Recuperado el 23 de mayo de 2016, de http://www.irena.org/menu/index.aspx?mnu=cat\&PriMenuID=44\&CatID=112.

29 IRENA, "Intellectual Property Rights: The Role of Patents in Renewable Energy Technology Innovation”, IRENA Working Paper, junio de 2013, pp. 15, 21.

${ }^{30}$ MEYER, “Global...” cit., p. 340.

${ }^{31}$ MEYER, “Epistemic...” cit., pp. 16-17.
} 
Estas últimas incluyen las organizaciones que recaban información científica y tecnológica y la aplican a un problema específico, aunque no creen derecho de forma $\operatorname{directa}^{32}$.

El carácter epistémico de la Agencia puede observarse en las distintas actividades que el Estatuto le encomienda, ya que todas ellas están destinadas a mejorar el conocimiento de prácticas, tecnologías y otros aspectos relativos a las energías renovables, así como a la eficiencia y la maximización de resultados a través del buen uso de tal conocimiento. En efecto, la Agencia nace con la intención de convertirse en un centro de excelencia en materia de tecnología de las energías renovables que promueva la gobernanza informada desde un punto de vista científico, al facilitar información creíble y útil a los reguladores ${ }^{33}$. No solo los gobiernos son los potenciales beneficiarios de las actividades de la Agencia; en este sentido, para los agentes privados relacionados con la planificación y realización de inversiones en infraestructuras de energías renovables, la información de la IRENA tiene un gran potencial de reducir sus costes transaccionales ${ }^{34}$.

\section{Descripción general de las actividades de la Agencia}

El primer grupo de actividades de la Agencia consiste en analizar, supervisar y sistematizar las prácticas actuales en materia de energías renovables ${ }^{35}$. En esencia, se trata de una labor de observación, estudio y seguimiento de las medidas, estrategias y políticas aplicadas en materia de energía renovables para así obtener información de calidad sobre las mejores prácticas. La labor de observación y seguimiento se completa con una actividad adicional de ordenación del conocimiento existente. Ahora bien, la Agencia no tiene competencias para establecer obligaciones para sus miembros ${ }^{36}$. Por lo tanto, en el supuesto de que la Agencia identifique una práctica especialmente útil para un Estado miembro, no podrá obligarlo a implementarla en su ámbito doméstico, pues

\footnotetext{
${ }^{32}$ Ibidem, p. 17.

${ }^{33}$ IRENA, Work Programme... cit., p. 6.

${ }^{34}$ MEYER, “Global...” cit., p. 339.

${ }^{35}$ Artículo IV.A.1.a) del Estatuto IRENA.

${ }^{36}$ Idem.
} 
el Estatuto se lo prohíbe expresamente. Más aún, no está previsto que la IRENA sea un foro para negociar la adopción de ese tipo de obligaciones ${ }^{37}$.

Un segundo grupo de actividades se refiere a la interacción con otras organizaciones internacionales y no gubernamentales, así como con redes públicas activas en el ámbito de las energías renovables. El objetivo es que la Agencia y el resto de organizaciones se beneficien mutuamente de sus experiencias y conocimientos. La Agencia basa sus relaciones con otras instituciones en dos ideas básicas: ser un complemento a lo que otros hacen, evitando el solapamiento de actividades; y aprovechar su carácter intergubernamental y universal para posicionarse como la institución internacional de referencia en la materia ${ }^{38}$. Por lo que se refiere a la comunicación y el intercambio de información con las Naciones Unidas, sus agencias especializadas y otras organizaciones internacionales, la IRENA tiene una presencia permanente en Nueva York y viene trabajando desde el año 2010 en el establecimiento en Viena de una oficina de enlace ${ }^{39}$.

Una tercera categoría de actividades de la IRENA es la prestación de servicios de asesoría y apoyo en materia de políticas energéticas a los miembros que los soliciten, incluyendo el asesoramiento en cuanto a financiación de las energías renovables ${ }^{40}$. La Agencia aspira a ser la organización de referencia a la que los Estados recurran cuando tengan interés en conocer los desarrollos más recientes en el diseño de políticas, marcos normativos, acuerdos institucionales y mecanismos para la financiación o las tecnologías relacionadas con las energías renovables ${ }^{41}$.

Otro grupo de actividades de la IRENA consiste en mejorar los mecanismos de transferencia de conocimientos y tecnología, así como el fomento del desarrollo de capacidades y competencias en los Estados miembros ${ }^{42}$.

Por último, el impulso a la investigación, la divulgación de información de normas técnicas sobre energías renovables y el fomento de la toma de conciencia pública acerca

\footnotetext{
${ }^{37}$ MEYER, “Global...” cit., p. 338.

${ }^{38}$ IRENA, Decision regarding the Work Programme and Budget for 2011. IRENA Doc. A/1/DC/8, 10 de julio de 2011, pp. 5-6. [En lo sucesivo, Work Programme and Budget 2011].

${ }^{39}$ IRENA, Decision on the 2010 Work Programme and Budget. IRENA Doc. IRENA/PC.3/dc.6, 17 de enero de 2010, p. 27. IRENA, Work Programme and Budget 2014-2015, cit., p. 47.

${ }^{40}$ Artículo IV.A.1.c) y f) del Estatuto IRENA.

${ }^{41}$ IRENA, Work Programme and Budget 2011, cit., p. 6.

${ }^{42}$ Artículo IV.A.1.d) del Estatuto IRENA.
} 
del potencial que ofrecen las energías renovables también forman parte de las actividades de la Agencia $^{43}$. Es importante mencionar que la IRENA no realiza investigación científica de forma directa, sino que se limita a sistematizar, transmitir y difundir los conocimientos existentes ${ }^{44}$.

\section{Principales iniciativas y proyectos de la Agencia}

Las iniciativas y los proyectos de la Agencia son de naturaleza transversal, inciden en dos o más objetivos y categorías de las actividades antes descritas. A continuación se describen algunos de los proyectos e iniciativas más emblemáticos de la Agencia.

\subsection{La evaluación del potencial para el despliegue de energías renovables}

Las necesidades energéticas crecen inexorablemente ligadas al aumento de la población y obligan a una respuesta por parte de los gobiernos en forma de inversiones y adopción de políticas. Desde la IRENA se está trabajando para que esta respuesta sea informada y tome en cuenta el potencial de las energías renovables. En ese sentido, un aspecto clave son los proyectos de "Evaluación del potencial para el despliegue de energías renovables" (Renewables Readiness Assessment - RRA). En este marco, la Agencia ha realizado estudios individuales completos de las condiciones para el despliegue de energías renovables en más de dieciocho países africanos, a los que ha ayudado a identificar su potencial y los ha asesorado en el desarrollo de políticas energéticas y de marcos normativos que les permitan aprovecharlo ${ }^{45}$.

\subsection{La función de nodo de confluencia para las energías renovables}

En el 2011, el secretario general de las Naciones Unidas lanzó la iniciativa Energía Sostenible para Todos (SE4ALL, por sus siglas en inglés), que aspira a lograr tres metas para el año 2030: a) generar acceso a la energía para todos; b) duplicar la ratio de eficiencia energética; y c) duplicar la contribución de las energías renovables a las

\footnotetext{
${ }^{43}$ Ibidem, artículo IV.A.1.g).

${ }^{44}$ MEYER, “Epistemic..." cit., p. 41.

45 IRENA. (2016). Renewables Readiness Assessment (RRA). Recuperado el 23 de mayo de 2016, de http://www.irena.org/menu/index.aspx?mnu=Subcat\&PriMenuID=35\&CatID=110\&SubcatID=164.
} 
fuentes globales de energía ${ }^{46}$. A la IRENA se le atribuyó el papel de ser el "nodo de confluencia de las energías renovables" (SE4ALL Renewables Hub) en el marco de esta iniciativa. La Agencia ya ha puesto en marcha el proyecto REmap2030 para elaborar la hoja de ruta dirigida a lograr el tercero de los objetivos de la iniciativa ${ }^{47}$. La Agencia ha estado trabajando con 26 países, que representan tres cuartos del consumo global de energía, para determinar las opciones y los costes estimados del despliegue acelerado de las energías renovables en el escenario de las inversiones globales en el sector de la energía previstas hasta el año 2030. Las primeras conclusiones apuntan a que existe la posibilidad de lograr duplicar la contribución de las energías renovables a las fuentes globales de energía sin coste alguno e incluso permitir ahorros económicos, siempre y cuando se adopten las medidas adecuadas ${ }^{48}$. De forma complementaria, la IRENA se ha propuesto desarrollar recomendaciones sobre políticas y suministrar servicios de asesoría técnica en el marco de uno de los programas bandera de la iniciativa SE4ALL conocido como Oportunidades de Alto Impacto (High Impact Oportunities) y establecer un marco de colaboración con el Banco Mundial, entre otras instituciones ${ }^{49}$.

\subsection{El Portal de Conocimiento sobre Energías Renovables}

El potencial de las energías renovables no se podrá aprovechar de forma eficiente a menos que los gobiernos y los inversores dispongan de información fiable sobre el funcionamiento de los mercados en los países o regiones de su interés, así como del tipo de políticas energéticas existentes, fuentes de financiamiento, tecnología disponible, etc.

Como ya hemos señalado antes, la aspiración principal de la IRENA es posicionarse como centro de excelencia de información sobre energías renovables. Para lograrlo, la IRENA está trabajando en la creación del Portal de Conocimiento sobre Energías Renovables (Knowledge Gateway), cuyo objetivo es permitir el libre acceso a través de internet a una amplia gama de información, actual y fácilmente localizable, sobre energías renovables como estadísticas de mercado, recursos potenciales, oportunidades

\footnotetext{
${ }^{46}$ NACIONES UNIDAS, Sustainable Energy for All: A Vision Statement by Ban Ki-Moon, SecretaryGeneral of the United Nations, Naciones Unidas, Nueva York, 2011, p. 4.

${ }^{47}$ IRENA. (2016). REmap2030 - A Renewable Energy Roadmap. Recuperado el 23 de mayo de 2016, de http://irena.org/remap/.

${ }^{48}$ IRENA, REmap2030. Hoja de ruta para las energías renovables: Resumen de las conclusiones, IRENA, Abu Dabi, 2014, p. 11 y ss.

${ }^{49}$ IRENA, Work Programme and Budget 2014-2015, cit., p. 10.
} 
de educación y laborales, costes relacionados con la innovación y la tecnología, etc. ${ }^{50}$ Otro ejemplo de colaboración interesante es la base de datos compartida entre la IRENA y la AIE con información sobre políticas y medidas en materia de energías renovables de 106 países $^{51}$.

El Portal también alojará el Atlas Global sobre Energías Renovables, que es la base de datos global más amplia y completa sobre el potencial que diferentes partes del mundo poseen para generar energía renovable y que actualmente ya se encuentra en funcionamiento aportando información sobre energía eólica y solar ${ }^{52}$. La Agencia está trabajando para incluir la información sobre el potencial de otras cuatro fuentes de energía renovable: la bioenergía, la geotérmica, la hidroeléctrica y la de los océanos ${ }^{53}$.

También merece ser destacada la información sobre el coste real de las energías renovables que la IRENA facilita tras haber analizado 8.000 proyectos sobre energías renovables llevados a cabo en 2013 en diferentes partes del mundo. Estos estudios solventan en parte las lagunas de conocimiento y permiten avanzar hacia la solución de uno de los principales obstáculos para el despliegue de este tipo de energías como es el miedo o mito de los altos $\operatorname{costes}^{54}$. Actualmente, la IRENA tiene planes para crear la Alianza sobre el Coste de las Renovables, una red global de información y análisis de $\operatorname{costes}^{55}$.

Esta información tiene un enorme potencial de dar impulso a las inversiones y es de gran utilidad para la adopción de planes energéticos nacionales y regionales.

\subsection{Iniciativas para promover las inversiones y el crecimiento económico}

Desde la IRENA se está intentando desmentir el mito de que las inversiones en energías renovables no son rentables y se trabaja para destacar los beneficios directos e indirectos que las inversiones han generado en la práctica, por ejemplo, sobre la

\footnotetext{
${ }^{50}$ Ibidem, p. 16.

${ }^{51}$ IEA/IRENA. (2016). Joint Policies and Measures database. Recuperado el 23 de mayo de 2016, de http://www.iea.org/policiesandmeasures/renewableenergy/.

52 IRENA. (2016). Global Atlas for Renewable Energy. Recuperado el 23 de mayo de 2016, de http://globalatlas.irena.org/.

${ }^{53}$ IRENA, Work Programme and Budget 2014-2015, cit., pp. 18-19.

${ }^{54}$ IRENA. (2016). COSTS: Renewable Energy Costs, Technologies and Markets. Recuperado el 23 de mayo de 2016, de http://costing.irena.org/

${ }^{55}$ IRENA, Work Programme and Budget 2014-2015, cit., p. 21.
} 
creación de empleos, el valor industrial añadido, la sostenibilidad ambiental o la generación de beneficios. La Agencia también identifica las barreras a las inversiones, así como los posibles mecanismos para reducir los riesgos asociados a este tipo de inversiones ${ }^{56}$.

Diversas iniciativas tienen por objetivo promover las inversiones y el crecimiento económico. Por ejemplo, la Agencia realiza análisis de políticas a nivel nacional y tiene previsto realizar análisis regionales en América Latina y el Caribe, así como en las regiones del Consejo de Cooperación del Golfo. También analiza las opciones de financiamiento a las que pueden recurrir los miembros o los inversores ${ }^{57}$.

Otras iniciativas regionales destacables incluyen el Corredor de Energía Limpia de África, con el que se pretende desarrollar un sistema de interconexión de energía ${ }^{58}$, así como el Corredor de Energía Limpia de América Central ${ }^{59}$.

Adicionalmente, la Agencia coopera con el Fondo para el Desarrollo de Abu Dabi para apoyar proyectos en los países en vías de desarrollo concediendo créditos concesionales por un monto no superior a los 350 millones de dólares. Un aspecto interesante es que los proyectos financiados se centran en aquellos que favorecen el proceso de aprendizaje y poseen además la cualidad de ser fácilmente replicados ${ }^{60}$.

Aunque formalmente la IRENA las trata como temas particulares, se pueden englobar en esta categoría la iniciativa Red Global de Energías Renovables para Islas (Global Renewable Energy Islands Network -GREIN-) y la Plataforma de Aprendizaje de Energías Renovables (Renewable Energy Learning Platform -IRELP-), actualmente en funcionamiento, que proporciona información sobre la oferta formativa y práctica.

\footnotetext{
${ }^{56}$ Ibidem, p. 24.

${ }^{57}$ Ibidem, pp. 26-27.

${ }^{58}$ IRENA, Working Together to Build and East and Southern African Clean Energy Corridor, IRENA, Abu Dabi, 2013.

${ }^{59}$ IRENA. (2015). Leaders Advance Plans to Unlock Renewable Energy Potential in Central America. Recuperado el 23 de mayo de http://www.irena.org/News/Description.aspx?NType=A\&mnu=cat\&PriMenuID=16\&CatID=84\&News_I $\mathrm{D}=395$.

${ }^{60}$ IRENA, Work Programme and Budget 2014-2015, cit., p. 27.
} 


\section{LOGROS Y RETOS FUTUROS DE LA AGENCIA}

La valoración de la Agencia en sus primeros años de existencia es, en su mayor parte, positiva. De hecho, si continúa en la trayectoria actual, el futuro de la organización es prometedor. A continuación describiremos algunos de sus logros principales.

\section{Los logros}

\subsection{Ser una organización internacional independiente}

Los Estados, antes de crear una organización internacional, muestran tendencia a reformar las estructuras institucionales existentes para dar cabida a los nuevos desafíos, incluso si tales instituciones no son eficientes o idóneas para impulsar la cooperación necesaria ${ }^{61}$. En ese sentido, antes que crear la IRENA como institución independiente, una opción hubiera sido reforzar la AIE, que ha venido trabajando con energías renovables desde hace años. Sin embargo, en virtud de su limitada membrecía, de los pocos recursos financieros y humanos que dedica a las energías renovables y de su compromiso ideológico con la energía nuclear y fósil, esta opción nunca fue considerada seriamente por los fundadores de la Agencia ${ }^{62}$. De hecho, algunas organizaciones internacionales como las Naciones Unidas, la OPEP y la AIE veían con desconfianza las propuestas de creación de la IRENA y algunas de ellas incluso se opusieron en los momentos previos a su establecimiento. Desde las Naciones Unidas la idea de crear una nueva organización fuera de sus marcos institucionales se veía como una crítica a sus actividades; desde la AIE, como un signo de desconfianza hacia ella; y desde la OPEP, como un peligro para los intereses económicos de sus industrias petroleras y de gas ${ }^{63}$. Así pues, en tal contexto, el nacimiento de la IRENA como organización internacional independiente debe ser reconocido como un logro en sí mismo ${ }^{64}$.

\footnotetext{
${ }^{61}$ VAN DE GRAAF, “Fragmentation...” cit., pp. 17 y 18.

${ }^{62}$ La AIE ha sido criticada por publicar proyecciones incorrectas sobre el potencial de las energías renovables para enfatizar la dependencia a largo plazo respecto de la energía nuclear y fósil. Véase VAN DE GRAAF, "Fragmentation..." cit., pp. 25-26.

${ }^{63}$ MEYER, "Global..." cit., p. 336.

${ }^{64}$ URPELAINEN y VAN DE GRAAF, “The International...”, cit., s/n.
} 


\subsection{Haber alcanzado una membrecía prácticamente universal}

La IRENA reconoce como uno de sus valores principales su amplia membrecía ${ }^{65}$. Desde su creación en abril de 2011, el número de miembros se ha duplicado. Como ya hemos mencionado, actualmente cuenta con 147 miembros y otros 29 Estados están en proceso de adhesión ${ }^{66}$. Así, la Agencia habrá alcanzado una membrecía universal en un período muy corto de tiempo. Esta situación no puede interpretarse sino como un voto de confianza en el potencial de la Agencia para generar oportunidades de inversión y conocimientos técnicos útiles para el crecimiento económico sostenible.

\subsection{Resultados con alto potencial económico a bajo coste}

La IRENA es una organización internacional con un presupuesto modesto. Las contribuciones de los miembros, que constituyen el presupuesto ordinario, apenas suman unos 22 millones de dólares al año. Los EAU realizan contribuciones voluntarias adicionales por 4,1 millones de dólares, que se destinan a actividades de gestión y administrativas de la Agencia, y, por su parte, Alemania contribuye voluntariamente con 4,9 millones dólares para el CIT. Así, para el año 2016 el presupuesto de la Agencia, contando el presupuesto ordinario y las contribuciones voluntarias, es de poco más de 30 millones de dólares ${ }^{67}$. Estos números se encuentran muy lejos del presupuesto de 62 millones de euros para 2016 de la Organización Internacional para la Energía Atómica $(\text { OIEA })^{68}$. Por otro lado, el presupuesto de la IRENA se aproxima un poco más al presupuesto de la AIE para 2016, que es de 27,4 millones de euros ${ }^{69}$. Sin embargo, mientras que la AIE tiene solo 29 miembros, la Agencia cuenta con 147.

\footnotetext{
${ }^{65}$ IRENA, Work Programme and Budget 2014-2015, cit., p. 44.

66 IRENA. (2016). Membership. Recuperado el 23 de mayo de 2016, de http://www.irena.org/menu/Index.aspx?mnu=Cat\&PriMenuID=46\&CatID=67.

${ }^{67}$ IRENA, Work Programme and Budget for 2016-2017: Report of the Director General. IRENA Doc. A/6/4, 17 de enero de 2016, p. 70. [En lo sucesivo, Work Programme and Budget 2016-2017].

68 IAEA. (2016). Regular Budget for 2016. Recuperado el 23 de mayo de 2016, de https://www.iaea.org/about/budget.

69 IEA. (2016) Organization and Structure. Recuperado el 23 de mayo de 2016, de $<$ http://www.iea.org/aboutus/faqs/organisationandstructure/>.
} 
Pese a las limitaciones presupuestarias, la Agencia ha sido capaz de implementar proyectos e iniciativas con bajo coste operacional pero con un gran potencial de generar beneficios económicos para miembros e inversores y ambientales para toda la comunidad internacional. Aunque en mayor o menor grado todas las actividades de la Agencia buscan este objetivo, destacan particularmente los trabajos sobre el coste real de las energías renovables, el Atlas Global sobre Energías Renovables y los RRA, a los que ya hemos hecho referencia previamente.

Si bien es verdad que no existen análisis científicos que cuantifiquen los beneficios económicos y ambientales que se derivan de las actividades de la IRENA, es evidente que la información que facilita en forma de opciones de inversión, tecnologías disponibles y potencial para el despliegue de las energías renovables en las distintas áreas geográficas reduce los costes de transacción de las inversiones en el sector y las hace más atractivas ${ }^{70}$. Más aún, aunque la generación de energía renovable aumenta rápidamente, en particular gracias a los avances tecnológicos y la reducción de costes, se trata de un sector en el que la información es escasa, a veces no es precisa o se encuentra viciada por intereses diversos ${ }^{71}$. Dado que la información fiable y neutral con frecuencia no está disponible, las actividades de la Agencia cobran especial valor.

\subsection{Ha sabido evitar la parálisis sobre las acciones globales sobre el cambio climático}

La resistencia de algunos países a asumir obligaciones cuantificables de reducción de gases de efecto invernadero ha sido la causa de que el régimen post-Kioto tardara tanto en llegar. De hecho, los compromisos nacionales voluntarios son el instrumento central del Acuerdo de París que guiará la lucha contra el cambio climático a partir de 2020. Como destacan URPELAINEN y VAN DE GRAAF, uno de los principales éxitos de la IRENA ha sido saber mantenerse al margen de los debates sobre las acciones que los países miembros deben llevar a cabo para hacer frente a la amenaza del cambio climático, debates que paralizan a la comunidad internacional ${ }^{72}$. El enfoque de la Agencia ha sido partir de la base de que las energías renovables son algo que todos

\footnotetext{
${ }^{70}$ Véase MEYER, “Global...” cit., pp. 319-347.

${ }^{71}$ IRENA, Work Programme and Budget 2014-2015, cit., p. 16.

72 URPELAINEN y VAN DE GRAAF, “The International...” cit., pp. 159-177; WIRTH, T. E. y DASCHLE, T. A., "A Blueprint to End Paralysis Over Global Action on Climate", Yale Environment 360,19 de mayo de 2014.
} 
aceptan como positivo, sin intentar justificar que deben preferirse a otras formas de energía. De este modo, trabaja exclusivamente para aportar información científica y técnica de calidad sobre las energías renovables, sin pretender la adopción de porcentajes de energías renovables cuantificables y obligatorios en la cesta de fuentes de energía de sus miembros ${ }^{73}$. De hecho, y como ya hemos mencionado, la Agencia no tiene competencias para fijar obligaciones para sus miembros ${ }^{74}$. Esta característica, lejos de hacer de la Agencia una institución débil, favorece una amplia participación, como demuestra su membrecía, prácticamente universal. Por otro lado, la Agencia no se presenta como una organización internacional que trabaja para resolver los problemas del cambio climático, aunque sus actividades sin duda son parte de la solución.

\section{Los retos de la Agencia}

En esta sección nos ocuparemos de los principales retos que debe afrontar la Agencia para lograr sus objetivos.

\subsection{Posicionarse como centro de información de excelencia a nivel global}

La Agencia aún no ha logrado suficiente visibilidad, por lo que sigue sin cumplirse su aspiración principal de posicionarse como centro de información de referencia a nivel global sobre las energías renovables. La IRENA es consciente de ello y una de las acciones para avanzar hacia este objetivo es contar con una publicación anual insignia ${ }^{75}$. En el 2014 publicó la primera edición de REthinking Energy, que contiene análisis y visiones de futuro con los que pretende informar a los encargados de elaborar políticas y estimular el debate sobre aspectos clave de las energías renovables ${ }^{76}$. En ese sentido, el carácter epistémico de la IRENA debe dar frutos de excelencia en REthinking Energy para poder situarla entre otras publicaciones sobre energía que gozan de trayectoria y reconocimiento internacional como Global Energy Outlook(GEO), que la AIE publica anualmente desde 1994 y que se ha convertido en la publicación más reconocida sobre

\footnotetext{
${ }^{73}$ URPELAINEN y VAN DE GRAAF, “The International...” cit., pp. 159-177.

${ }^{74}$ MEYER, “Global...” cit., p. 338.

${ }^{75}$ RÖHRKASEN y WESTPHAL, “IRENA: Stay...” cit., p. 3.

${ }^{76}$ IRENA, Rethinking Energy 2014: Towards a New Power System, IRENA, Abu Dabi, 2014.
} 
análisis de los mercados de energía y sus tendencias globales, galardonada con diversos premios por su calidad ${ }^{77}$.

Por otro lado, como hemos mencionado antes, a la IRENA se le atribuyó el papel de ser el "nodo de confluencia de las energías renovables" en el marco de la iniciativa Energía Sostenible para Todos (SE4ALL). La Agencia debe aprovechar esta oportunidad para ganar visibilidad e influir en el debate global sobre las energías renovables ${ }^{78}$.

\subsection{Lograr movilizar el apoyo de países clave que aún no son miembros}

En el ámbito de las energías renovables, la investigación científica en materia de energías renovables está dominada por un grupo muy pequeño de países, de ahí la importancia de lograr la participación de todos ellos. Por ejemplo, el $80 \%$ de las patentes sobre energías renovables están en manos de seis países: Estados Unidos, Japón, Alemania, Francia, Corea del Sur y el Reino Unido. Por su parte, Brasil y China son los protagonistas en el mundo en vías de desarrollo ${ }^{79}$. La IRENA puede sentirse satisfecha de que ha conseguido atraer a prácticamente todos ellos como miembros. Sin embargo, la Agencia ha fallado a la hora de movilizar la cooperación de países estratégicos como Brasil, que aún no es miembro, o Canadá, el único miembro de la AIE aún ausente en la IRENA ${ }^{80}$.

Brasil se encuentra entre los cinco países con mayor capacidad instalada para generar energía renovable ${ }^{81} \mathrm{y}$, además de producir cerca del $45 \%$ de su energía por fuentes renovables, es líder en biocombustibles: exporta el 48\% de etanol a nivel global y es el segundo mayor productor de biodiésel ${ }^{82}$. Brasil puede contribuir significativamente a las

\footnotetext{
77 IEA. (2016). About WEO. Recuperado el 23 de mayo de 2016, de http://www.worldenergyoutlook.org/aboutweo/.

${ }^{78}$ IRENA, Work Programme and Budget 2014-2015, cit., p. 64.

${ }^{79}$ MEYER, “Epistemic..." cit., p. 38.

${ }^{80}$ VAN DE GRAAF, T., "How IRENA is Reshaping Global Energy Architecture", European Energy Review, 29 de marzo de 2012.

${ }^{81}$ REN21, Renewables 2014: Global Status Report, REN21 c/o UNEP, París, 2014, p. 26.

82 IRENA, "Global Bioenergy: Supply and Demand Projections", IRENA Working Paper for Remap 2030, septiembre de 2014, pp. 16, 17.
} 
actividades de la Agencia, en particular en el ámbito de la innovación sobre energías renovables en sectores como el hidrológico, el marino y el de los biocombustibles ${ }^{83}$.

La razón que ha impedido a Brasil ser parte de la IRENA ha sido, según ha reconocido su director general, una idea equivocada de que la Agencia está en contra de la energía hidroeléctrica, los biocombustibles y la biomasa ${ }^{84}$. Estas fuentes de energía son controvertidas pues en ocasiones se asocian con efectos negativos de tipo social y ambiental. Por ejemplo, una planificación inadecuada puede conducir a primar la producción de biocombustibles en detrimento de la producción de alimentos, a la deforestación y a la desertificación, mientras que la construcción de infraestructuras hidroeléctricas afecta a los ecosistemas y los ciclos naturales de los ríos ${ }^{85}$.

Por lo que se refiere a Canadá, el haberse mantenido al margen de la Agencia le ha valido críticas internas por mirar hacia otro lado en un momento clave de transición energética y por perder oportunidades comerciales y de crecimiento ${ }^{86}$.

\subsection{Evitar su instrumentalización}

La Agencia ha vivido momentos difíciles, en particular por las interferencias de sus miembros en el funcionamiento de algunos de sus órganos y por la falta de compromiso de algunos de ellos con los objetivos de la IRENA. Evitar su instrumentalización, es decir, ser utilizada por algunos miembros para lograr objetivos particulares, se presenta como una de las prioridades de la política institucional de la IRENA.

Como ya hemos mencionado, la idea de que se creara la Agencia no era del agrado de algunas organizaciones internacionales como la ONU, la AIE y la OPEP, que la percibían como competencia o crítica a su desempeño. La idea tampoco ilusionaba a los países miembros que ejercen un rol dominante en esas instituciones, ya que temían perder protagonismo en el ámbito de las energías renovables, ni a los que les

\footnotetext{
83 Por ejemplo, Brasil tiene el mayor número de patentes en tecnología para producir energía aprovechando los océanos. UNEP, UCTSD, EPO, Patents and Clean Energy: Bridging the Gap between Evidence and Policy: Final Report, Naciones Unidas, Suiza, 2010, pp. 9, 21, 31. Resaltando que Brasil, por su competitividad, es uno de los principales receptores de tecnología a través de patentes, véase IRENA, "Intellectual..." cit., p. 17.

${ }^{84}$ VAN DE GRAAF, “How IRENA..." cit.

${ }^{85}$ PRING, G. et al., “The Impact...” cit., pp. 13-37.

${ }^{86}$ CLEAN ENERGY CANADA. (2014). Tracking the Energy Revolution: Global Edition 2014, p. 17. Recuperado el 23 de mayo de 2016, de www.cleanenergycanada.org.
} 
preocupaba que la creación de la Agencia pudiera afectar a los intereses comerciales de sus industrias productoras de petróleo o gas ${ }^{87}$. Aunque la mayor parte de los miembros de esas instituciones son también miembros de la IRENA — con la excepción de Canadá y Venezuela, que son, respectivamente, miembros fundadores de la AIE y de la OPEP - , se han cuestionado las motivaciones que llevaron a algunos países a ingresar en la Agencia e implícitamente se les ha asociado con algunos de los problemas que afectaron a su funcionamiento tras su creación.

En efecto, el primer año y medio de vida de la Agencia fue muy turbulento, como refleja el hecho de que la que fuera directora general interina, la francesa Hélène Pelosse, decidiera renunciar tan solo quince meses después de haber asumido el cargo, que en principio tenía una duración de cuatro años ${ }^{88}$. Tras su dimisión explicó que los EAU habían pedido a Francia que la hiciera renunciar por intentar impulsar la paridad de género dentro de la Agencia ${ }^{89}$. Por otro lado, un miembro del comité administrativo de la IRENA apuntó que Pelosse había cometido algunas equivocaciones como manifestarse en la página web de la Agencia en contra de la captura y almacenamiento de carbono, algo que en su opinión no podía hacer la directora de una organización internacional, o demorarse en la adopción del programa de trabajo y en la contratación de directores para llevarlo a cabo ${ }^{90}$. Sin embargo, Scheer sostuvo que la mayoría de los problemas que afrontó Pelosse eran consecuencia de la falta de apoyo de los países miembros. La demora en la adopción del programa de trabajo la atribuyó a que muchos países retuvieron sus contribuciones, lo que limitó el margen de acción de Pelosse. En su opinión, no todos los miembros tomaban en serio a la Agencia y algunos parecían haber ingresado en ella principalmente para influir en su programa de trabajo ${ }^{91}$.

El director general que sucedió a Pelosse, Adnan Z. Amin, goza de mayor respaldo de los miembros y muchos de los problemas descritos parecen haberse superado: las contribuciones al presupuesto ordinario han dejado de ser de naturaleza voluntaria y los programas de trabajo se han adoptado puntualmente y con objetivos acordes con el mandato original de la Agencia. Así, aunque muchos países pudieron verse motivados a

\footnotetext{
${ }^{87}$ MEYER, "Global..." cit., p. 336.

${ }^{88}$ STANTON, CH., "IRENA Head Quits", The National, 20 de octubre de 2010.

${ }^{89}$ KEMPF, H., “J'ai été contrainte de démissionner", Le Monde, 29 de octubre de 2010.

${ }^{90}$ POWERS, D. S., "Budget and Leadership Problems Plague Renewable Energy Agency", New York Times, 29 de noviembre de 2010.

${ }^{91}$ Idem.
} 
acceder a la institución para influir en el desarrollo de su agenda, de momento no hay nada que indique que esto se esté produciendo en la práctica ${ }^{92}$. Sin embargo, la Agencia debe trabajar para que esto siga así.

\section{CONCLUSIONES}

En el año 2009, el secretario general de las Naciones Unidas sostuvo ante la Asamblea General que el cambio climático es el mayor desafío que afronta la humanidad ${ }^{93}$. También dijo que, para evitar consecuencias catastróficas para las personas y el planeta, la comunidad internacional tenía menos de diez años para detener el incremento en las emisiones de gases de efecto invernadero ${ }^{94}$. Eso fue hace siete años, pero, lejos de detenerse, las emisiones siguen aumentando globalmente ${ }^{95}$. En efecto, pese a la magnitud de este desafío, las respuestas de la comunidad internacional siguen haciéndose esperar. La IRENA, pese a ser una organización internacional muy pequeña, tiene un gran potencial de contribuir significativamente a la transición hacia patrones energéticos sostenibles. Su enfoque, basado en transmitir información fiable, neutral, gratuita y universal sobre las opciones existentes, no futuras, para el despliegue de diversas formas de energías renovables, es una vía muy eficaz e inteligente para motivar las inversiones y las políticas nacionales y regionales. La IRENA no desperdicia energía en debates estériles que intenten justificar la apuesta por las energías renovables. Por el contrario, aprovecha el funcionamiento natural de la iniciativa privada, siempre dispuesta a implicarse en actividades rentables. También es especialmente adecuado el enfoque de la IRENA para un sistema internacional en el que cuesta construir la solidaridad entre Estados y en el que los intereses egoístas de algunos de ellos fundamentan muchas de sus decisiones. Su diseño, su enfoque y el contexto en el que debe funcionar indican que la IRENA es un acierto de innovación institucional. Su papel como catalizador del despliegue de las energías renovables hace de la IRENA un elemento indispensable de la gobernanza global de las energías. Por lo tanto, es

\footnotetext{
92 MEYER, “Global...” cit., p. 336.

93 SECRETARIO GENERAL BAN KI-MOON. (2009, 10 de agosto). Remarks at the $39^{\text {th }}$ Plenary Assembly of the World Federation of United Nations Associations. Recuperado el 23 de mayo de 2016, de http://www.un.org/apps/news/infocus/sgspeeches/statments_full.asp?statID=555\#.VUjELfntlBc.

94 Ibidem.

95 IPCC, "Resumen..." cit., p. 6; OLIVIER, J. et al., Trends in Global CO2 Emissions: 2014 Report, Netherlands Environmental Assessment Agency, La Haya, 2014.
} 
aconsejable que el sector académico y también el público le reconozcan su función como un elemento relevante ante los desafíos energéticos del siglo XXI.

\section{FUENTES Y BIBLIOGRAFÍA}

ABBOTT, K. W., "The Transnational Regime Complex for Climate Change", Environment and Planning C: Government and Policy, vol. 30, núm. 4, 2012, pp. 571-590.

BUTLER, J., “The Second Earth Summit”, Geography Review, mayo de 2003.

CLEAN ENERGY CANADA (2014), Tracking the Energy Revolution: Global Edition 2014.

CORRELJÉ, A. y VAN DER LINDE, C., "Energy Supply Security and Geopolitics: A European Perspective", Energy Policy, vol. 34, núm. 5, 2006, pp. 532-543.

ESCRIBANO, G., "Fragmented Energy Governance and the Provision of Global Public Goods", Global Policy, vol. 6, Issue 2, 2015, pp. 97-106.

IPCC, "Resumen para responsables de políticas", EDENHOFER, O. et al. (eds.), Cambio climático 2014: Mitigación del cambio climático, Cambridge University Press, Cambridge, Reino Unido y Nueva York, NY, Estados Unidos de América, 2015.

IRENA, Work Programme and Budget for 2014-2015: Report of the Director General. IRENA Doc. A/4/3, 18 de enero de 2014.

IRENA, "Intellectual Property Rights: The Role of Patents in Renewable Energy Technology Innovation”, IRENA Working Paper, junio de 2013, pp. 4-32.

IRENA, "Global Bioenergy: Supply and Demand Projections", IRENA Working Paper for Remap 2030, septiembre de 2014.

KEMPF, H., “J'ai été contrainte de démissionner”, Le Monde, 29 de octubre de 2010.

LONG, A., "Complexity in Global Energy-Environment Governance", Minnesota Journal of Law, Science \& Technology, vol. 15, 2014, pp. 1059-1115.

MEYER, T., "Global Public Goods, Governance Risk, and International Energy”, Duke Journal of Comparative \& International Law, vol. 22, 2012, pp. 319-347.

MEYER, T., "Epistemic Institutions and Epistemic Cooperation in International Environmental Governance”, Transnational Environmental Law, vol. 2, núm. 1, 2013, pp. 15-44.

OLIVIER, J. et al., Trends in Global CO2 Emissions: 2014 Report, Netherlands Environmental Assessment Agency, La Haya, 2014.

POWERS, D. S., "Budget and Leadership Problems Plague Renewable Energy Agency", New York Times, 29 de noviembre de 2010.

PRING, G. et al., "The Impact of Energy on Health, Environment, and Sustainable Development", ZILlMAN, Donald N. et al. (eds.), Beyond the Carbon Economy: Energy in Transition, Oxford University Press, Nueva York, 2008, pp. 13-37.

REN21, Renewables 2014: Global Status Report, REN21 c/o UNEP, París, 2014.

RÖHRKASTEN, S. y WESTPHAL, K., "IRENA: Stay the Course", SWP Comments, núm. 37, noviembre de 2012, pp. 1-4.

RÖHRKASTEN, S. y WESTPHAL, K., "IRENA and Germany Foreign Renewable Energy Policy: Aiming at Multilevel Governance and an Internationalization fo the Energiewende?", SWP: Working Paper FG 8, 2013/01, septiembre de 2013.

STANTON, CH., “IRENA Head Quits”, The National, 20 de octubre de 2010. 
UNEP, UCTSD, EPO, Patents and Clean Energy: Bridging the Gap between Evidence and Policy: Final Report, Naciones Unidas, Suiza, 2010.

URPELAINEN, J. y VAN DE GRAAF, T., "The International Renewable Energy Agency: A Success Story in Institutional Innovation?", International Environmental Agreements: Politics, Law and Economics, vol. 15, núm. 2, 2015, pp. 159-177.

VAN DE GRAAF, T., "Fragmentation in Global Energy Governance: Explaining the Creation of IRENA”, Global Environmental Politics, 2013, vol. 13, núm. 3, pp. 14-33.

VAN DE GRAAF, T., "How IRENA is Reshaping Global Energy Architecture”, European Energy Review, 29 de marzo de 2012.

WRIGHT, G., "The International Renewable Energy Agency: A Global Voice for the Renewable Energy Era?", Renewable Energy Law and Policy Review, vol. 4, 2011, pp. 251-268. 